\title{
The Limits of Simplicity: Toward Geocomputational Honesty in Urban Modeling
}

In GeoDynamics, P. Atkinson, G. foody, S. Darby, F. Wu (Eds.) Florida: CRC Press. (2004)

\author{
Keith C. Clarke \\ Department of Geography/NCGIA \\ University of California, Santa Barbara \\ Santa Barbara, CA, USA. \\ Telephone: 1-805-893-7961 \\ FAX: 1-805-893-3146 \\ Email: kclarke@geog.ucsb.edu
}

\begin{abstract}
Occam's razor informs us that simpler models are better. Yet models of geographical systems must often be complex to capture the richness of the human and natural environments. When critical decisions, such as those affecting human safety, depend on models, the model must be sufficient for the task, yet can become impossible for all but the modeler to understand holistically. This paper outlines five model components, and examines how a move toward simplicity impacts each, focussing on the critical stage of model calibration. Three model applications to the same data are examined in terms of how their calibrations took place. A comparison of the results shows that model outcomes are subjective, and more shaped by their role in decision-making than by scientific criteria. Model reduction, a goal as yet lacking methods, may therefore not be a useful way forward for geocomputational modeling until the full context of models and the ethics of modeling are better understood.
\end{abstract}

\section{Introduction}

A model is an abstraction of an object, system or process that permits knowledge to be gained about reality by conducting experiments on the model. Constraining modeling to that of interest to the geographer, a model should abstract geographic space and the dynamic processes that take place within it, and be able to simulate spatial processes usefully. At the absurd extreme, a model could be more complicated than the data chosen to represent the spatio-temporal dynamics in question. Occam's razor tells us, of course, that when two models are of equal explanatory power, the simpler one is preferred. Einstein stated that "a model should be as simple as possible and yet no simpler." Some earth systems are so predictable that they are indeed amenable to modeling with the simplest of models. For example, the drop in atmospheric pressure as a function of elevation is so predictable as to be suitable for building a measuring instrument for the latter using the former as a proxy. Of course few human systems, or coupled human/environmental systems are this simple. Geography's past is laced with attempts to use overly simple predictive or explanatory models for human phenomena, the gravity model being a good example. In some cases, the simplicity these models offer serves to produce insight, or at least fill textbooks, in the absence of geographic laws. In this paper, I raise the issue of model simplicity, and discuss Einstein's question of how much simplicity is enough. 


\section{Simplicity in Models}

The problem of model simplicity is that models have multiple components and are often complicated. Complex systems theory has shown that even simple models can produce complexity and chaos when given the right initial conditions or rules, though the theory gives little guidance on how to invert this sequence. Formally, models are sets of inputs, outputs, and algorithms that duplicate processes or forms. The more complicated the process or form, the less simple a model can be.

Models have been thought of as consisting of four components. These are (1) input, both of data and parameters, often forming initial conditions; (2) algorithms, usually formulas, heuristics, or programs that operate on the data, apply rules, enforce limits and conditions, etc.; (3) assumptions, representing constraints placed on the data and algorithms or simplifications of the conditions under which the algorithms operate; and (4) outputs, both of data (the results or forecasts) and of model performance such as goodness of fit. Considering modeling itself requires the addition of a fifth component, that of the modeler, and including their knowledge, specific purpose, level of use, sophistication, and ethics. By level of use, we distinguish between the user who seeks only results (but wants credible models and modelers), the user who themselves run the model, the user who seeks to change the model, and the user who seeks to design a model. We term the former two secondary users, since the model is taken as given. The latter two are primary users, and include modelers.

Communication between primary and secondary users usually consists of model documentation and published or unpublished papers, manuals, or websites.

Spatial inputs and outputs are usually maps, and the algorithms in geography and geographic information science usually simulate a human or physical phenomenon, changes in the phenomenon, and changes in its cartographic representation. Modeling itself, however, is a process. A modeler builds data or samples that describe the inputs, selects or writes algorithms that simulate the form or process, applies the model, and interprets the output. Among those outputs are descriptions of model performance, forecasts of unknown outputs, and data sets that may be inputs to further models.

Others have sought answers to the simplicity/complexity paradox, although not always from the context of urban modeling. Smith (1997) for example, examined the treatment of simplicity versus complexity evident in a series of papers on population forecasting. Smith noted that "It is not necessary that simpler models be more accurate than complex models to be useful” (Smith, 1997, p. 559). Long (1995) defined three types of complexity (model specification, degree of disaggregation, and assumptions/alternatives), and Smith argued that these could be used to classify models by their complexity in each area. He concluded that "There is a substantial body of evidence, then, supporting the conclusion that more complex models generally do not lead to more accurate forecasts of total population than can be achieved with simpler models” (Smith, 1997, p. 560-1). He pointed out other criteria than accuracy as being important, and noted that complex models can make the modeler appear sophisticated, "whereas the use of simple models and techniques may make him/her appear to be stupid, lazy, or poorly trained (Smith, 1997, p. 563). Occam's razor seems to have many dimensions!

Why might it be necessary to seek the limits of simplicity? I illustrate why using a practical example of immense environmental significance in the United States, that of the Yucca Mountain high level radioactive waste disposal site in Nevada. The problem requiring modeling is that of finding a permanent, safe and environmentally suitable storage location for the thousands of tons of highly radioactive waste from decades of civilian and military work in nuclear energy. The specifications of this site are that it should remain "safe" for at least 10,000 years. Safety is defined by the level of radionuclides at stream gaging stations a few kilometres downstream from the location. Failure in this case could be at least nationally 
catastrophic, and safety depends on the waste remaining dry. The recommendation to bury the waste deep underground dates back to 1957. The selection of the Yucca Mountain site came after a very lengthy process of elimination, debate over safe storage and studies by the regulatory agencies and the National Academy of Science. Scientists at the Los Alamos National Laboratory in the Earth and Environmental Sciences division took on the task of using computer simulation to approach the problem of long term stability at the site.

Simple risk assessment methodology uses probability distributions on the random variables and perhaps Monte Carlo methods to gain knowledge of the overall probability of failure. As the models become more complex, so also does the probability density function. In 1993 a study reporting the detection of radionuclides from Cold War era surface nuclear testing in the deep subsurface at Yucca Mountain introduced the immense complexity of modeling subsurface faults and so-called "fast hydrographic pathways" (Fabryka-Martin et al., 1993). With the earliest, more simple, models the travel times of radionuclides to the measurement point was about 350,000 years. Adding Dual Permeability, allowing rock fracture, generated a new and far more complex model that showed travel times to only tens to hundreds of years, thereby failing the acceptance criterion. Although more complex, these models are more credible because they can duplicate the data on the fast hydrographic pathways.

Nevertheless, highly complex models become cumbersome when dealing with the public decision making process. Robinson (2003) notes that for a numerical model to be useful in decision making, it must be correct and appropriate to the questions beings asked from the point of view of the scientists and model developers, and understandable and believable from the perspective of decision makers. A report noted that “...the linkage between many of the components and parameters is so complex that often the only way to judge the effect on calculated results of changing a parameter or a submodel is through computational sensitivity tests.” (NWTRB, 2002). The latest model, called TSPA-LA, has nine major subcomponent models with nine interacting processes, and a total of 70 separate models, each with multiple input and complex interconnectivity. Some components are apparently similar (e.g. Climate inputs and assumptions), but interact in complex ways (in this case through unsaturated zone flow and saturated zonal flow and transport). Some submodels produce outputs that enter into macro-level models directly, while in other cases the same values are inputs for more models, such as those related to disruptive events.

In such a case, does any one person comprehend the entire model, even at a superficial level? How can it be proven that one or more variables actually contributes to the explanatory power of the model? More important, how can scientists establish sufficient credibility in such a complex model that sensible (or even any) political decisions are possible, especially in a highly charged decision-making environment? Los Alamos scientists have undertaken a process of model reduction as a consequence. Model reduction is the simplification of complex models through judicious reduction of the number of variables being simulated. Methods for model reduction do not yet exist, and deserve research attention. For example, when does a complex model pass the layman understandability threshold, and when it does, how much of the original model's behavior is still represented in the system? What variables can be made constant, and which eliminate or assumed away altogether? How much uncertainty is introduced into a model system by model reduction? In statistics, reducing the sample size usually increases the uncertainty. Is the same true at the model level? What are the particularly irreducible elements, perhaps the primitives, of models in which geographic space is an inherent component? Such questions are non-trivial, but beg attention if we are to work toward models that hold the credibility of their primary and secondary users.

\section{Honesty in Models and Modeling}

Sensitivity testing is obligatory to establish credibility in complex models. Sensitivity tests 
are usually the last stage of model calibration. The modeler is also usually responsible for calibrating the model, by tinkering with control parameters and model behavior, and at least attempting to validate the model. While validation may actually be impossible in some cases (Oreskes et al, 1994), calibration is obviously among the most essential obligations of modeling. Model calibration is the process by which the controlling parameters of a model are adjusted to optimize the model's performance, that is the degree to which the model's output resembles the reality that the model is designed to simulate. This involves necessarily quantitative assessments of the degree of fit between the modeled world and the real world, and a measurement of the model's resilience, that is, how sensitive it is to its input, outputs, algorithms, and calibration. That the obligation to make these assessments is that of the modeler is part of what the authors of the Banff Statement termed "Honesty in modeling" (Clarke et al., 2002, p. 296). Honest and transparency are coincident, as Myers and Kitsuse stated "The heart of the problem lies in the secrecy with which the forecaster enters assumptions and prepares his models” (Myers and Kitsuse, 2000).

Such an approach could be considered an essential component of modeling ethics generally, and even more so in Geography where the real world is our target. Uncalibrated models should have the status of untested hypotheses. Such is not often the case, however. Even reputable peer reviewed journals occasionally publish papers on models that are only conceptual, are untested, or tested only on synthetic or toy data sets. These papers are often characterized by statements such as "a visual comparison of the model results", or "the model behavior appears realistic in nature.” Assumptions go unlisted, limitations are dismissed, data are used without an explanation of the lineage or dimensions, and fudge factors sometimes outnumber model variables and parameters. A common practice is to use values for parameters that are "common in the literature", or looked up from an obscure source. The Earth's coefficient of refraction for the atmosphere, for example, has long been assumed to be 0.13 , a value derived from the history of surveying. This number is a gross average for a value known to vary from 0.0 to 1.0 and even beyond these limits under unusual circumstances, such as in a mirage.

While simplicity in model form is desirable, indeed mandatory, what about simplicity in the other four components of modeling? Simplicity is inherent in the model assumptions, as these are often designed to constrain and make abstract the model's algorithms and formulas. Simplicity is not desirable in inputs, other than in parameter specification, since the best data available to the scientist, in their judgement, should always be that used. Nevertheless, what data are used as inputs is often determined by the processing capabilities and the project's budget. Data output is often also complex, and requires interpretation, although our own work has shown that simple visualizations of model output can be highly effective, for example, as animations. Part of data output is performance data. Should there be simplicity in calibration and sensitivity testing?

Reviews of the literature in urban growth modeling (e.g. Agarwal et al., 2000; Wegener, 1994; EPA, 2000), show that precious few models are even calibrated at all, let alone validated or sensitivity tested. Most common is to present the results of a model and invite the viewer to note how "similar" it is to the real world. Such a lack of attention to calibration has not, apparently, prevented the model's widespread use. Doubts are often assumed away because the data limitations or tractability issues exceed these as modeling concerns.

If one uses the argument that we model not to predict the future, but to change it, then the above concerns are perhaps insignificant. The development of new models obviously requires some degree of trial and error in model design. Models are also testbeds, experimental environments, or laboratories, where conceptual or philosophical issues can be explored (e.g. McMillan, 1996). This is often the case with games, in which some models are intended for education or creative exploration. Such uses are similar to the link between 
cartography and visualization; or science and belief. These uses are not, strictly, of concern in science. Science based models have to work in and of themselves. Methods for model calibration include bootstrapping and hind casting. If models are to be used for decisions, to determine human safety in design, or to validate costly programs at public expense, then clearly we need to hold models to a more rigorous, or at least more valid, standard. Such issues are of no small importance in geographical models, and in geocomputation at large. Even minor issues, such as random number generation (Van Niel and Leffan, 2003), can have profound consequences for modeling. Whether or not model algorithms and code are transparent is at the very core of science: without repeatability there can be no proof of spurious results, falsification, or progress toward theory.

\section{A Case Study}

The simplicity question can be partially restated as: what is the minimum scientifically acceptable level of urban model calibration? Whether this question can be answered in general for all models is debatable. A complete ethics code for modeling is obviously too broad for a single paper. The domain of concern will be urban models and issues of their calibration and sensitivity testing. This discussion examines the problem in the context of a set of three models as applied to Santa Barbara, California. The three models are the SLEUTH model (Clarke et al., 1997; Clarke and Gaydos, 1998), the What If? Planning model by Richard Klosterman (2001), and the SCOPE model (Onsted, 2002).

Using the case study approach, the three models are analyzed in terms of their components, their assumptions, and their calibration processes. As a means of further exploring the consequences of modeling variants, their forecasts are also compared and the spatial dissimilarities explored in the light of the data for Santa Barbara.

\subsection{The SLEUTH Model}

SLEUTH is a cellular automaton (CA) model that simulates land use change as a consequence of urban growth. The CA is characterized by working on a grid space of pixels, with a neighborhood of eight cells of two cell states (urban/non urban), and five transition rules that act in sequential time steps. The states are acted upon by behavior rules, and these rules can self modify to adapt to a place and simulate change according to what have been historically the most important characteristics. More details about the SLEUTH model can be found in the chapters by Dietzel and by Goldstein in this volume.

SLEUTH requires five GIS-based inputs: urbanization, land use, transportation, areas excluded from urbanization, slopes, and hillshading for visualization. The input layers must have the same number of rows and columns, and be geo-referenced accurately. For statistical calibration of the model, urban extent must be available for at least four temporal snapshots, that for four dates, terminating with the most recently available map. Urbanization results from a "seed" urban file with the oldest urban year, and at least two road maps that interact with a slope layer to allow the generation of new nuclei for outward growth. Besides the topographic slope, a constraint map represents water bodies, natural and agricultural reserves. After reading the input layers, initializing random numbers and controlling parameters, a predefined number of interactions take place that correspond to the passage of time. A model outer loop executes each growth history and retains statistical data, while an inner loop executes the growth rules for a single year.

The change rules that will determine the state of each individual cell are taken according to the neighborhood of each cell, those rules are: 1. Diffusion, 2. Breed, 3. Spread, 4. Slope Resistance, and 5. Road Gravity. A set of self-modification rules also control the parameters, allowing the model to modify its own behavior over time, controlled by constants and the measured rate of growth. This allows the model to parallel known historical conditions by 
calibration, and also to aid in understanding the importance and intensity of the different scores in a probabilistic way. The land use model embedded within SLEUTH is a second phase after the growth model has iterated for a single pass, and uses the amount of urban growth as its driver. This model, termed the deltatron (Candau and Clarke, 2000), is tightly coupled with the urbanization model. The dynamics of the land cover change are defined through a four-step process in which pixels are selected at random as candidate locations and changed by a series of locally determined rules controlled largely by proximity and the land cover class transition matrix. Forecasts can be for either urban growth alone (Figure 1), or for land cover forecasts and their associated uncertainty (Candau, 2000a).

SLEUTH is public domain C-language source code, available for download online. It is not suitable for systems other than UNIX or its variants. A sample data set (demo_city) is provided with a complete set of calibration results. A full set of web-based documentation is on line at http://www.ncgia.ucsb.edu/projects/gig.

\subsection{SLEUTH Calibration}

SLEUTH calibration is described in detail in Clarke et al. (1996) and Silva and Clarke (2002). This "brute force" process has been automated, so that the model code tries many of the combinations and permutations of the control parameters and performs multiple runs from the seed year to the present (last) data set, each time measuring the goodness of fit between the modeled and the real distributions. Results are sorted, and parameters of the highest scoring model runs are used to begin the next, more refined sequences of permutations over the parameter space. Initial exploration of the parameter space uses a condensed, resampled and smaller version of the data sets, and as the calibration closes in on the "best" run, the data are increased in spatial resolution. Between phases in the calibration, the user tries to extract the values that best match the five factors that control the behavior of the system. Coefficient combinations result in combinations of thirteen metrics: each either the coefficient of determination of fit between actual and predicted values for the pattern (such as number of pixels, number of edges, number of clusters), for spatial metrics such as shape measures, or for specific targets, such as the correspondence of land use and closeness to the final pixel count (Clarke and Gaydos, 1998). The highest scoring numeric results from each factor that controls the behavior of the system from each phase of calibration feed the subsequent phase, with user-determined weights assigned to the different metrics. Calibration relies on maximizing spatial and other statistics between the model behavior and the known data at specific calibration data years. Monte Carlo simulation is used, and averages are computed across multiple runs to ensure robustness of the solutions.

Recent work on calibration has included testing of the process (Silva and Clarke, 2002), and testing of the temporal sensitivity of the data inputs (Candau, 2000b). Candau's work showed that short term forecasts are most accurate when calibrated with short term data. Silva and Clarke showed that the selection process for the "best" model results is itself an interpretation of the forces shaping urbanization in a region. Recent work on Atlanta (Yang and Lo, 2003) has shown that the calibration process steps that take advantage of assumptions of scale independence are invalid, meaning that ideally the calibration should take place exclusively at the full spatial resolution of the data. Discussion of these findings has sometimes taken place on the model's on-line discussion forum. In this book, the chapter by Dietzel examines the impact of changing the geographic extent of the spatial units on the results of the SLEUTH calibration process. Similarly, Goldstein's chapter examines the substitution of a genetic algorithm for the brute force method that SLEUTH uses. Both are contributions very much in accordance with furthering honesty in modeling by critically examining how the model is applied, and by testing variations in model results that are the consequence of how the input data are aggregated and applied, rather than by parameter control.

\subsection{The SCOPE model}


The South Coast Outlook and Participation Experience (SCOPE) model was originally based on the UGROW model (EPA, 200, p. 128), adapted and rebuilt in PowerSim and eventually rebuilt again at UCSB by graduate student Jeffrey Onsted in the STELLA modeling language (HPS, 1995; Onsted, 2002). SCOPE is a systems dynamics model in the Forrester tradition (Forrester, 1969). This type of model posits simple numerical relations between variables that can be simulated with basic equations that link values. STELLA allows these empirical relations to be represented in a complex but logically consistent graphic system, that uses icons and a basic flow diagram to build the model (Figure 2). Variables can be stocks and flows, with measurable interactions with finite units, or factors influencing rates and flows but not contributing to them directly.

The SCOPE model consists of hundreds of variable-to-variable links, expressed by equations. Each of these involves its own set of assumptions. These assumptions can be documented in the model code itself, and in SCOPE's case are also contained in the model's documentation. The complete set of individual assumptions is immense, and many assumptions are simply minor variants on relations and rates separated for one of the five economic sectors in the model (population, quality of life, housing, businesses and jobs, and land use). The housing sector is further divided into low, middle and upper income, students and senior citizens. In essence, a separate model is maintained for each, with some assumptions enforced to keep aggregates the sum of their parts. The model, when complete, can be used at several levels. At the modeler level, actual parameters and links can be modified and the program used to generate statistical output in the form of charts and tables. At the user level, the model has a STELLA interface that allows users to turn on and off aggregate impacts, such as the application of particular policies, enforcement of rules and zoning, and sets of assumptions leading to the particular scenarios being developed by the model's funders. This is done using graphical icons representing switches. At a third and higher level, this interaction can take place across the world wide web (http://zenith.geog.ucsb.edu). A last level uses only the scenarios developed through both this and the SLEUTH model to interact with integrated planning scenarios (http://zenith.geog.ucsb.edu/scenarios).

The SCOPE model is proprietary in that it was funded as a local not-for-profit project by the Santa Barbara Economic Community Project. Nevertheless, the model is on-line, and so can be examined in totality, as can the assumptions behind the model and formulas used. STELLA is a proprietary modeling environment, but is common in educational environments, and is subsidized for students.

\subsection{SCOPE Calibration}

SCOPE's original and secondary design were public participatory experiments. A series of meetings were conducted with various local stakeholder and citizens groups, including planners, local activists, the general public, and many others while the model was partially complete to solicit input on what variables were important and how they were best modeled. 
This was a long and arduous process, involving hundreds of hours of work, but the result was encouraging in that when users understood the model and its assumptions, they had high credibility in its forecasts, even when they were counterintuitive. For example, SCOPE's demographic module consistently projected population decline in the region over the next few years, a fact at first rejected and later accepted by the users.

Obviously no user and few modelers can understand the whole SCOPE model. Nevertheless, the highly graphic STELLA modeling language and its different user interfaces meant that all users could zoom into and get specific about critical parts of the model from their perspective. Separate workshops were eventually held to work separately on the major sectors of the model. Final integration was largely a guided trial and error undertaking, with the constraint that as one component changed a single calibration metric was used, that of the Symmetrical Mean Absolute Percent Error (SMAPE) (Tayman and Swanson 1996). Onsted (2002, p. 140-1) stated "We considered an output "validated", at least for now, if it had a SMAPE of less than 10\%." This type of calibration has been called "statistical conclusion validity", a type of internal validity, described by Cook and Campbell as "inferences about whether it is reasonable to presume covariation given a specified level and the obtained variances" (Cook and Campbell, 1979, p. 41). While such an approach is similar to bootstrapping, in SCOPE's case rather extensive sets of historical data on a large number of the attributes being simulated were available and could be used for SMAPE calculations. Continued refinements to the model have since improved on the original reported calibration results. Onsted (2002) conducted a sensitivity test of sorts, providing a comprehensive bias and error table, and considering the impacts of the errors on forecasts.

\subsection{What if?}

The What if? modeling and planning support system was developed by Richard Klosterman at the University of Akron. He describes the system as "an interactive GIS-based planning support system that supports all aspects of the planning process: conducting a land suitability analysis, projecting future land use demands, and evaluating the likely impacts of alternative policy choices and assumptions.” (Klosterman, 1997, p. 1). The suite of software that constitutes the model is built on top of ESRI's ArcGIS using MapObjects and Visual basic. The software is proprietary, and available from Klosterman's consultancy via their web site (http://www.what-if-pss.com).

For this experiment, a software license was obtained and the data for Santa Barbara, equivalent to the above project, compiled into the ArcView shape files necessary for What if? Data preparation in What if? consists of bringing into the software a large number of data files that contribute to the weighted selection by overlay of the vector polygons that meet various criteria. Initial preparation consists of selecting the layers, creating their union, and eliminating errors such as sliver polygons. This creates a set of Uniform Analysis Zones (UAZs) that are used as the granularity of analysis. Land suitability for urban use (high and low density residential, and commercial/industrial) are then computed using standard weighted overlay multicriterion decision making methods. The land suitability method was Rating and Weighting (RAW), using current land use, distances to roads, streams, the coastline and commercial centres, topographic slope, the 100 year floodplain, and preserving agricultural landuse. The user must select the weights, based on prior experience or intuition, and the result is a land suitability map (Figure 3). These maps find their way into an allocation routine that determines future demand for each use (based on exogenous estimates), and assigns development based on probabilities calculated from the weights. The result is a land suitability map, and maps showing development under different assumptions and scenarios about allocations. The software comes on a CD, which also contains data for a simulated data set (Edge City). 
The approach was applied to Santa Barbara and used for the land suitability stage only. The software distinguishes between drivers (the growth projection and demand) and factors (the land suitability and assessment, and the land supply). A third stage, allocation, brings demand and supply together. Thus our application used only part of the software, which is a full planning support system. Results are shown in figure 3. Considerable effort was placed into getting the same data as used in SCOPE and SLEUTH into the What if? simulation.

\subsection{What if? Calibration}

The What if? documentation contains no information at all about calibration. References are made to models that use the multicriterion weighted overlay method, such as CUF II (Landis and Zhang, 1998) and MEPLAN (Echenique et al., 1990). This tradition goes back to the work of McHarg and Lowry within planning, and is an established approach. The modeling, or rather forecasting, is an allocation of an exogenous demand using spatially determined input criteria such as buffers, thematic layers, exclusion areas, and other factors.

To be fair, the planning approach of What If? is based on assuming an invariant present and an almost absent past. This is because the model application here dealt with only the land suitability component of the multi-step What if? approach, so eliminating the past as a model input and dealing only with overlay of current data. In the application, past twenty-year decadal population totals were used to make linear projections of future population as part of the housing demand estimation, but these were totals only and not spatially disaggregated. Thus the intelligence passes from the explicit assumptions of the model to the skills and experience of the modeler in compiling accurate input data, in selecting and computing weights, and in combining the requisite layers effectively. Forecasting routines are more deterministic, and even the number of forecast periods is prescribed. The full model bases future projections on past trends and allows the user to modify future conditions. Consequently, every time the model is applied to a new area, its application is completely customized, and while reproducible, is rarely sufficiently documented that the same results would be attained even from the same data. This is because the What If? Approach is seen as a way of engaging participants in the scenrio-based planning process, a good example of what has been called "urban modeling as story telling” (Guharthakurta 2002). The model relies entirely on the credibility of the input data and the acceptability of the results. The actual code is compiled, and while the principles are demonstrated clearly in the documentation, the actual executable code is black-box.

\section{Model Comparisons}

Each of the models applied to Santa Barbara produced a forecast for the year 2030. It should be pointed out that these models were chosen as being representative of three classes of urban growth modeling strategies: cellular automata, systems dynamics, and rule-based allocation. Whether or not the results are also representative of these classes of models will be left for further work. There is considerable value in comparing models that use different strategies, particularly because agreement between models is often seen as an improvement in the reliability of the forecast, especially in weather and economic forecasting. Comparison between the models can only take the form of a matching of forecasts, since the actual future distribution and amount of housing in Santa Barbara in 2030 remains unknown. Thus we can "simply" compare the models to each other. One of the few values that is an estimate in common among the forecasts is the additional area of built up land. These directly comparable values are listed in table 1. 
It should be pointed out that even these forecasts have so many caveats as to be mostly incomparable. The What if? forecasts come from the "What if? growth report, generated by the software during forecasting. The value is a sum for separate numbers for low density, high density, medium density and multi-unit housing, plus commercial and industrial land. Of this area, far more is allocated to commercial/industrial than to residential by 8343.5 to 5662.8 hectares. It was unclear how applying an exogenous demand estimate would change these values. Spatially, commercial growth was allocated near existing areas of that land use and particularly in Carpinteria (furthest east) and Goleta (west). High density residential followed the same pattern, while low density residential was more scattered.

The SCOPE forecasts were generated by flipping the "base-line" policy switch, thus in a black-box fashion incorporating all of the general plan assumptions that go along with this prebuilt scenario. These are listed as follows: "The Baseline scenario assumes that the current urban limit line and other boundaries shall remain unchanged. Also, the South Coast will continue to build out as outlined in existing General Plans, Local Community Plans and per existing zoning. The market forces and demographics that drove change in the past will continue to influence the construction of new commercial and residential development. Infill will continue as it has along with redevelopment while large parcels will be more limited. There will be some extension of urbanized areas. The Gaviota coast, however (with the exception of Naples), shall be protected as well as the agricultural areas in the Carpinteria Valley.” As such, clearly a greater amount of local knowledge has been built into the forecast, even though we do not know explicitly what values and parameters have been set. Such information is, however, buried deep in the documentation.

For SLEUTH, the assumptions made are summarized as follows: “This scenario uses the most basic exclusion layer, and uses an additional 'Urban Boundary' GIS layer to constrain growth. The Urban Boundary data was derived through a series of workshops held by the Economic Forecasting Project of Santa Barbara where local stakeholders defined a desired spatial limit to urban expansion. All growth in this scenario is constrained within the urban boundary." This constraint is the obvious reason why this is the lowest of the three forecasts. Spatially, the growth is allocated as infill in Santa Barbara and Goleta, expansion in Gaviota and Carpinteria, and along a strip following US highway 101 west from Goleta toward Gaviota. Even the enforcement of the urban boundary, however, seems unlikely to stop urban sprawl.

While the model forecasts are at least comparable, they incorporate not only a new set of assumptions about future (rather than assumed algorithm and data) behavior, but also different ways of dealing with uncertainty. Since What If? was not used beyond the land suitability phase, no probabilistic boundaries on the spatial or non-spatial forecasts were given. Nevertheless, the precision of the predictions (the report rounds data to the nearest $1 / 10^{\text {th }}$ of a hectare) gives an impression of certainty. The predictions were given at decadal increments. SCOPE's forecasts are broad, rounded to the nearest 1,000 acres given the error estimates listed in the SMAPE tables. SCOPE does, however, generate annual amounts for the forecast values, allowing the declining rate of addition of new urban land to be quite visible. SLEUTH's forecasts were not forthcoming. Data from the annual forecast images were brought into a GIS for tabulation. SLEUTH had by far the most detailed spatial granularity (30 x 30 meter pixels), but the Monte Carlo treatment of uncertainty added an averaging quality to the estimates. The new urban areas were ranked by uncertainty. 1,606 ha of growth was deemed $95-100 \%$ certain, with the remainder distributed across the probabilities greater than $50 \%$. Maps of percent likelihood of urbanization for Monte Carlo simulations were computed by the SLEUTH program in its predict mode, and were 
available for consultation.

So which model's results are the "simplest?" All require further analysis, interpretation, and require deeper understanding of both the input data and the model. From the public's point of view, far more variation in informed decision-making has come from variants in graphical presentation of the results than in their statistical validity, level of calibration, or model robustness. It is not even evident that an overlay of the maps, or an averaging of the forecasts for specific targets is any more useful than the same taken independently. Only the passage of time, of course, can actually validate these forecasts. And in the meantime, especially with the SCOPE and SLEUTH models being used in devising a new community plan in the form of the Regional Impacts of Growth Study (SBRECP, 2003) of course the models themselves could--indeed are designed to--influence the result. Thus, Heisenberg's uncertainty principle places the model's use ever further from the scientific goals we set as ethical standards. Where we began with simple ethical directives with data and algorithms, as we move through the primary to the secondary model user, indeed on to the tertiary model "decision consumer", the science of modeling becomes more of an art, and one highly influenced by the methods used for division, simplification, and presentation of the modeling results. Perhaps foremost among urban planning uses in this respect is the use of scenarios as a modeling reduction tool. In each case, the model applications discussed here resorted to scenarios when communicating results. As a method in management science, scenarios indeed have a foundation in experience, yet this can hardly be claimed for Urban Planning and Geography (Xiang and Clarke, 2003).

\section{Discussion}

This chapter posed the question of what is the minimum scientifically acceptable level of urban model calibration? Obviously the minimum (and perhaps the median) current level of calibration is zero, irrespective of validation. Using the truth in labelling approach that has permeated the spatial data and metadata standards arena, an obvious conclusion to answer the question would be that all models should attempt to assess their internal consistency using whatever methods or statistical means that are suitable. The number of digits used to present numerical results should be rounded to levels appropriate to the expected variation, and at least some measure or estimate of variance should be made. At the extreme minimum, the lower base would be that of repetition. The modeler is obliged to say "I ran the experiment twice, and got the same (or different) results each time." Without this, the models are not scientific. At least some kind of report on calibration and sensitivity testing should accompany every model application too, and certainly if the work is to be peer reviewed.

In the examples and discussion, a whole new suite of meanings of "honesty in modeling" have emerged. With the five components of modeling, the primary model user has ethical obligations to the secondary and tertiary users. Thus, data need to be the best available to the scientist, accurate, timely and of the correct precision and spatial resolution; algorithms need to be rigorous, relevant, not-oversimplified and subjected to peer review; assumptions need to be relevant, logical and documented; and output needs to be thorough, sufficient to detect errors and suitable for use, and to include performance measures and success criteria for the model. This discussion introduced a fifth component, the primary modelers themselves. The modeler has the ultimate obligation to ensure that models are used appropriately, not only in the scientific sense of being accurate and correct, but in the sense that their outputs, reductions and simplifications will influence critical decisions, many involving human safety, most involving efficiency or satisfaction with human life. For these results, the modeler is equally to blame as the politician who acts upon them. 
In conclusion, simplicity is not always a desirada for models or modeling. Einstein's statement about how simple is profound. In this case, the challenge becomes one of reductionism. How simple can a complex model be made to become understandable? What is lost when such a model reduction is made? Most members of society would rather have an incomprehensible model that gives credible and useful results than an understandable model. Our own work has shown us that the general public seeks to place faith in modelers, and desires credible modelers as much as credible models. This, of course, places great responsibility on the modeler, indeed begs the more comprehensive set of ethical standards that today we instill in our graduate students only casually. We should not only think of ethics when legal problems arise (Wachs 1982). Furthermore, there are a whole suite of "more simple" reductionist methods that can be applied with minimal effort. Among these are attention to calibration, accuracy, repeatability, documentation, sensitivity testing, model use in decision-making, and information communication and dissemination. Modeling with spatial data is hardly in its infancy.

It has been pointed out that the views presented in this chapter assume that decisions regarding urban development are made scientifically, and that policy makers think according to the logic of scientific reasoning, and that this is a dubious assumption. There is little doubt that models, or at least modelers, have influenced urban and regional planning in the past. With increasing use of simulation in all aspects of human life (Casti, 1997), the flood of data now available for mapping of urban phenomena, and the ubiquitous availability of analysis tools such as GIS, it is likely that in the future we will see increasing faith placed by the general public in the models that become integrated into the planning process. At least once in the past, during the 1970s, urban modeling's progress was hampered, and even declared dead, because of a lack of attention to the seven sins (Lee, 1994). Like it or not, urban models have risen from the grave, and been given another chance to work, and work well. Simple models may be understandable to the world at large, but they may not do an adequate job of simulating the complexity of our world. At the same time, urban modelers should not just talk among themselves, regardless of how simple or complex, honest or dishonest their efforts. It is time that geoComputational models moved out from behind the curtain, and confronted the complex and complicated scientific world of geodynamics, in the understanding of which they are a useful part.

\section{Acknowledgements}

This work has received support from the NSF in the United States under the Urban Research Initiative (Project number 9817761). I am grateful to Dr. Bruce Robinson of Los Alamos National Laboratory for information and graphics concerning modeling at the Yucca Mountain Waste Repository. The SLEUTH model was developed with funding from the United States Geological Survey, and the SCOPE model with funds from the Irvine Foundation via the Santa Barbara Economic Community Project. The What If! Model was developed by Richard Klosterman, and applied to Santa Barbara by UCSB Geography undergraduate student Samantha Ying and graduate student Martin Herold. Thanks to the anonymous reviewers of this chapter for some thought-provoking comments, that have improved the final version.

\section{References}


AGARWAL, C., GREEN, G.L., GROVE, M., EVANS, T., and SCHWEIK, C., 2000, A review and assessment of land-use change models: Dynamics of space, time, and human choice. (Bloomington: US Forest Service and the Center for the Study of Institutions, Population, and Environmental Change [CIPEC])

CANDAU, J., 2000a. Visualizing Modeled Land Cover Change and Related Uncertainty. First International Conference on Geographic Information Science, Proceedings, GIScience 2000 (Savannah: http://www.giscience.org/GIScience2000/posters/235Candau.pdf)

CANDAU, J. 2000b. Calibrating a Cellular Automaton Model of Urban Growth in a Timely Manner. Proceedings (CD-ROM) 4th International Conference on Integrating GIS and Environmental Modeling (GIS/EM4). (Boulder: GIS/EM Secretariat)

CANDAU, J. and CLARKE, K. C. 2000. Probabilistic Land Cover Modeling Using Deltatrons. Proceedings (CD-ROM) , URISA 2000 Conference. (Orlando: Urban and Regional Information Systems Association)

CANDAU, J., RASMUSSEN, S. and CLARKE, K. C. 2000. Structure and Dynamics of a Coupled Cellular Automaton for Land Use/Land Cover Change. Proceedings (CDROM) 4th International Conference on Integrating GIS and Environmental Modeling (GIS/EM4). (Boulder: GIS/EM Secretariat)

CASTI, J. L. 1997. Would-be worlds: How simulation is changing the frontiers of science. (New York: J. Wiley)

CLARKE, K.C., HOPPEN, S., and GAYDOS, L. 1996. Methods and techniques for rigorous calibration of a cellular automaton model of urban growth. Proceedings (CD-ROM) Third International Conference/Workshop on Integrating Geographic Information Systems and Environmental Modeling. (Boulder: GIS/EM Secretariat)

CLARKE K.C., HOPPEN, S., and GAYDOS, L. 1997. A self-modifying cellular automata model of historical urbanization in the San Francisco Bay area. Environment and Planning B: Planning and Design, 24: 247-261.

CLARKE, K. C., and GAYDOS. L. J. 1998. Loose-coupling a cellular automation model and GIS: long-term urban growth prediction for San Francisco and Washington/Baltimore. International Journal of Geographical Information Science. 12, 7, 699-714.

CLARKE, K. C, PARKS, B. O., and CRANE, M. P. (Eds), 2002 Geographic Information Systems and Environmental Modeling (Upper Saddle River: Prentice Hall).

COOK, T.D. and CAMPBELL, D.T. 1979. Quasi-Experimentation. (Boston: Houghton Mifflin)

ECHENIQUE, M. H., FLOWERDEW, A. D., HUNT, J. D., MAYO, T. R., SKIDMORE, I. J. and SIMMONDS, D. C. 1990. The MEPLAN models of Bilbao, Leeds and Dortmund. Transport Reviews, 10, 309-22.

EPA, U. S. 2000. Projecting land-use change: A summary of models for assessing the effects of community growth and change on land-use patterns. (Cincinnati: U.S. Environmental Protection Agency, Office of Research and Development)

FABRYKA-MARTIN, J.T., WIGHTMAN, S.J., MURPHY, W.J., WICKHAM, M.P., CAFFEE, M.W., NIMZ, G.J., SOUTHON, J.R., and SHARMA, P., 1993. Distribution of chlorine-36 in the unsaturated zone at Yucca Mountain: An indicator of fast transport paths Proceedings, FOCUS '93: Site Characterization and Model Validation, Las Vegas, Nevada, 26-29 Sept. 1993.

FORRESTER, J. W. 1969. Urban Dynamics. (Cambridge: The M.I.T. Press)

GUHATHAKURTA, S. 2002. Urban modeling as storytelling: using simulation models as a narrative. Environment and Planning B, 29, 6, 895 - 911

HPS. 1995. STELLA: High Performance Systems. URL: http://www.hps-inc.com/.

KLOSTERMAN, R. E. 2001. The What if? Collaborative Planning Support System. In R. K. Brail and R. E. Klosterman, eds. Integrating Geographic Information Systems, models, and Visualization Tools. (Redlands: ESRI and New brunswick: Center for Urban Policy Research)

KLOSTERMAN, R. E. 1997 The What if? Collaborative Planning Support System, 
Proceedings, ESRI Annual Users Conference. http://gis.esri.com/library/userconf/proc97/abstracts/a305.htm

LANDIS, J. D., and ZHANG, M. 1998. The Second Generation of the California Urban Futures Model: Parts I, II, and III Environment and Planning B: Planning and Design. 25, 657-666, 795-824.

LEE, D.B. 1994. Retrospective on large-scale urban models. Journal of the American Planning Association, 60, $1,35-40$.

LONG, J. F. 1995. Complexity, accuracy, and utility of official population projections. Mathematical Population Studies, 5, 217-234.

McMILLAN, W. 1996. Fun and games: serious toys for city modelling in a GIS environment, in P.Longley and M.Batty (Eds.), Spatial Analysis: Modelling in a GIS Environment. (London: Longman Geoinformation) pp. 153-165.

MYERS, D. and KITSUSE, A. 2000. Constructing the future in planning:A survey of theories and tools. Journal of Planning Education and Research, 29, 221-231.

NWTRB 2002. NWTRB Letter Report of Congress and Secretary of Energy, January $24^{\text {th }}$, 2002. http://www.nwtrb.gov/reports/2002report.pdf

ONSTED, J. A. 2002. SCOPE: A Modification and Application of the Forrester Model to the South Coast of Santa Barbara County. MA Thesis, Department of Geography, University of California, Santa Barbara. http://zenith.geog.ucsb.edu/title.html

ROBINSON, B. 2003. Use of Science-based predictive models to inform environmental management: Yucca Mountain Waste Repository. CARE Workshop, UCSB. Unpublished presentation.

SBRECP. 2003. South Coast Regional Impacts of Growth Study. (Santa Barbara Region Economic Community Project: http://www.sbecp.org/documents.htm)

SMITH, S. K. 1997. Further thoughts on simplicity and complexity in population projection models, International Journal of Forecasting, 13, 557-565.

SILVA, E.A, and CLARKE, K.C. 2002. Calibration of the SLEUTH urban growth model for Lisbon and Porto, Portugal. Computers, Environment and Urban Systems, 26, 6, 525-552.

TAYMAN, J. and SWANSON, D. A. 1996. On The Utility of Population Forecasts. Demography, 33, 4, 523-528.

VAN NIEL, and LEFFAN, S. 2003.Gambling with randomness: the use of pseudo-random number generators in GIS International Journal of Geographical Information Science, 17, 1, $49-68$

WACHS, M. (1982) Ethical dilemmas in forecasting for public policy. Public Administration Review, 42. 562-567.

WEGENER, M. 1994. Operational urban models: State of the art. Journal of the American Planning Association, 60, 1, 17-30.

XIANG, W-N. and CLARKE, K. C. 2003 The use of scenarios in land use planning. Environment and Planning B, 30, 885-909.

YANG, X. and LO, C. P. (2003) Modeling urban growth and landscape change in the Atlanta metropolitan area, International Journal of Geographical Information Science, 17, 5, 463-488. 
FIGURE CAPTIONS

Figure 1: Forecast urban growth for Santa Barbara in 2030 using SLEUTH. Yellow was urban in 2000 . Red is urban with $100 \%$ probability in 2030 . Shades of green are increasing urban, starting at $50 \%$.

Figure 2: Sample STELLA code from the SCOPE model

Figure 3: What If? land suitability assessments for Santa Barbara

Table 1: Comparison of model results for additional developed area in Santa Barbara (2000-2030)

Figure 1

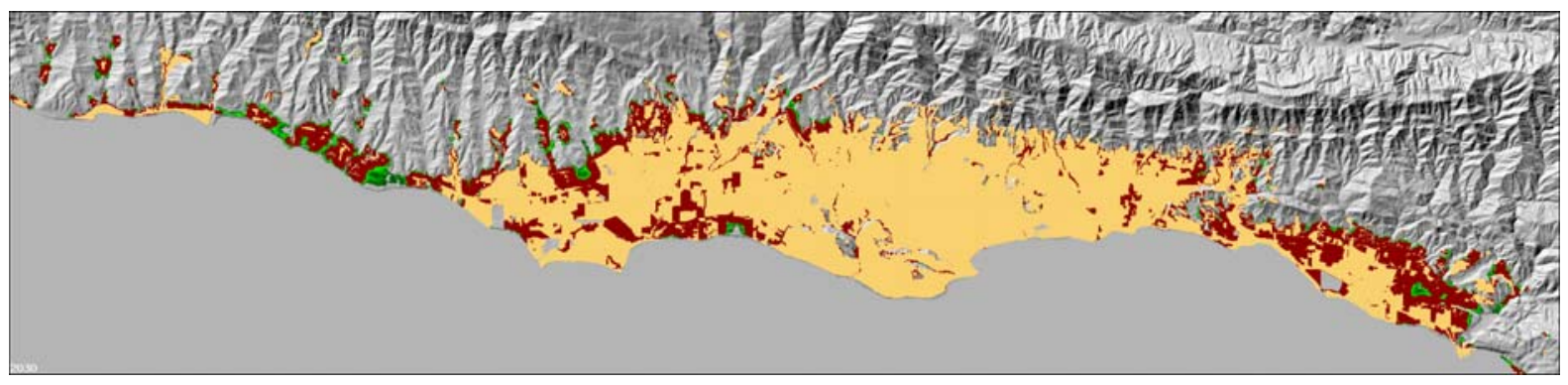

Figure 2

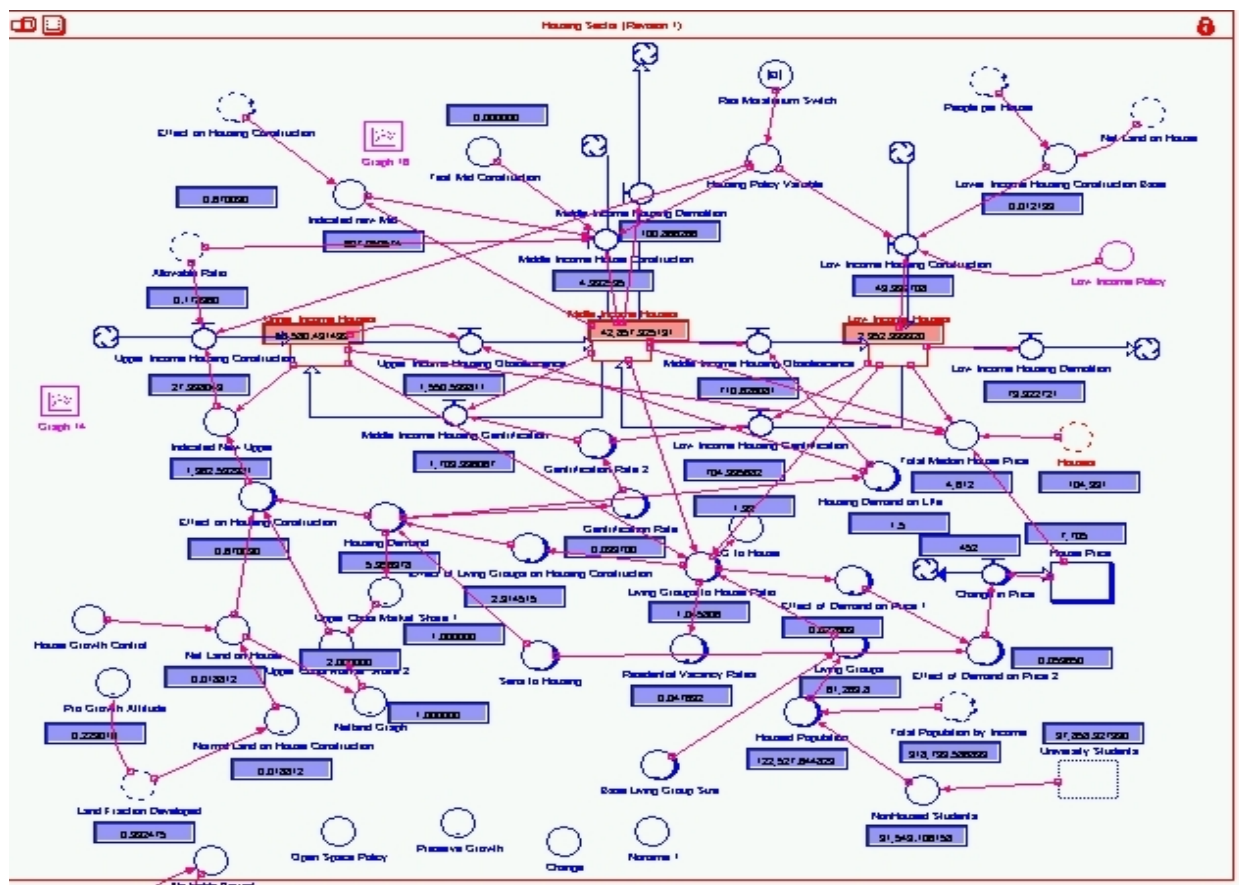


Figure 3

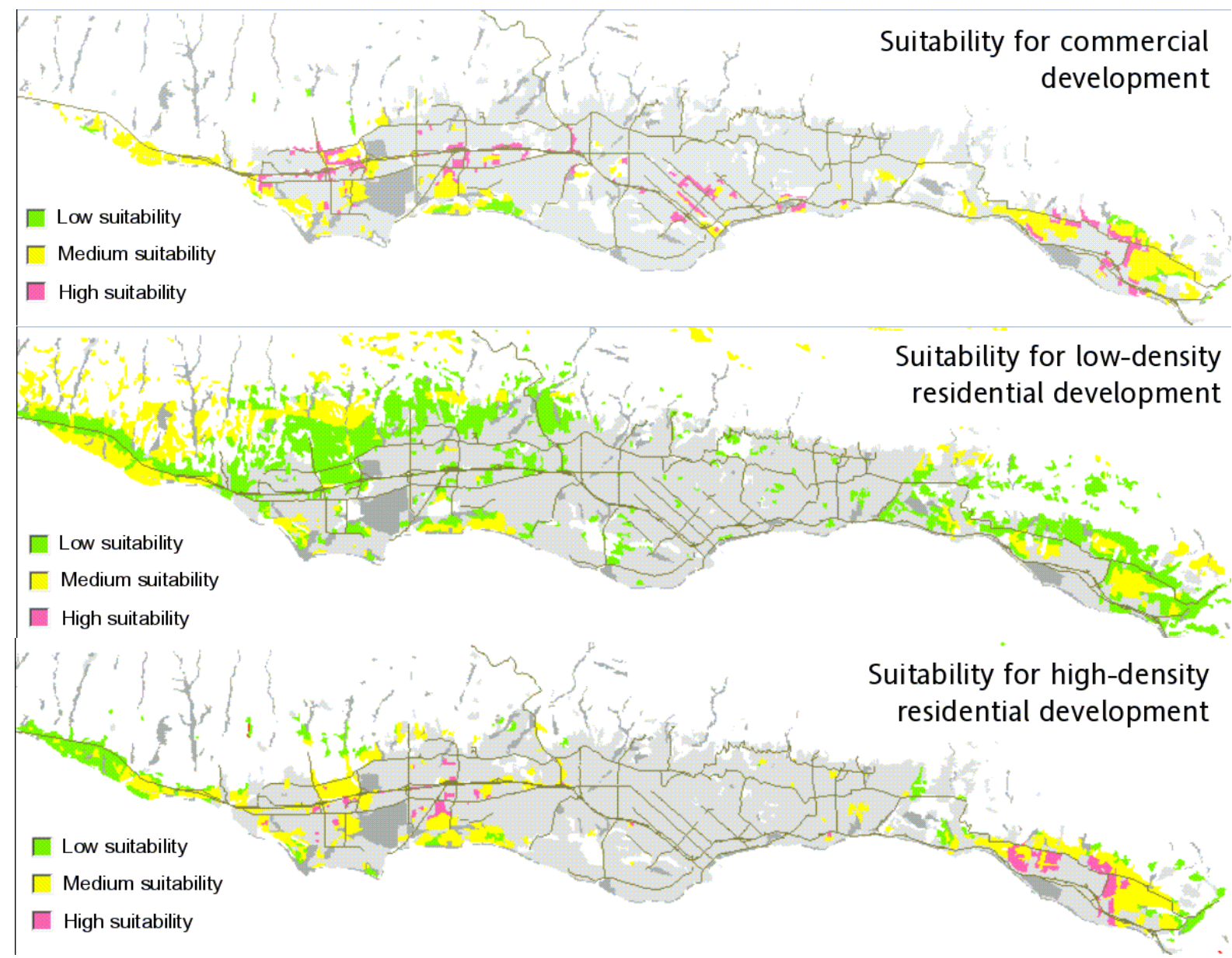

Table 1

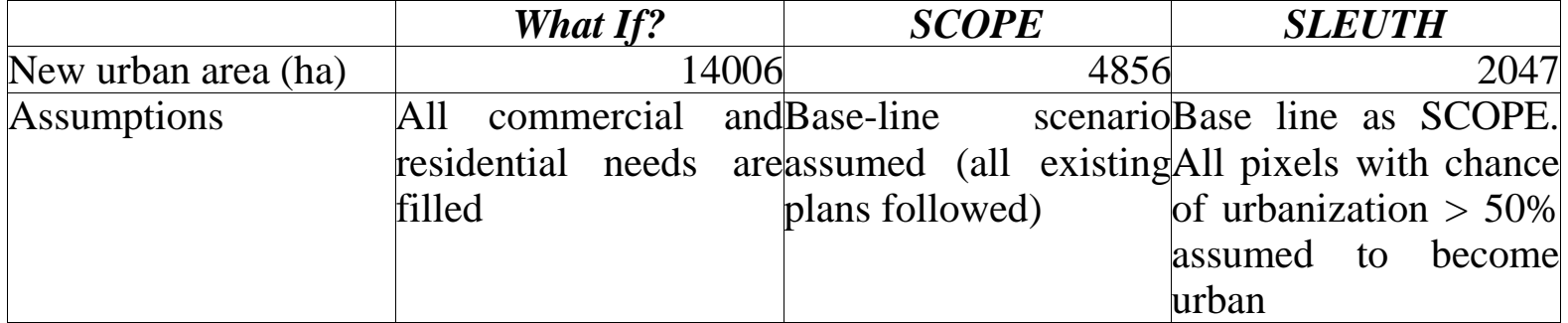

